\title{
Bayesian Adaptive B-spline Estimation in Proportional Hazards Frailty Models: Supplement
}

\author{
Emmanuel Sharef* and Robert L. Strawderman ${ }^{\dagger}$ \\ and David Ruppert* \\ Cornell University \\ Ithaca, NY 14853 \\ e-mail: ess28@cornell.edu; rls54@cornell.edu; dr24@cornell.edu \\ Mark Cowen $^{\ddagger}$ and Lakshmi Halasyamani ${ }^{\S}$ \\ St. Joseph Mercy Hospital \\ Ann Arbor MI 48106 \\ e-mail: cowenm@trinity-health.org; halasyal@trinity-health.org
}

\section{Contents}

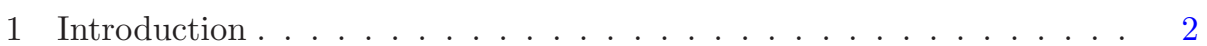

2 Estimation procedure . . . . . . . . . . . . . . 2

2.1 Additional notation . . . . . . . . . . . . . . . . 2

2.2 Obtaining initial values $\ldots \ldots \ldots \ldots \ldots$

2.3 Metropolis-Hastings MCMC steps . . . . . . . . . . . 5

2.3.1 Updating the frailties $U_{i} \ldots \ldots \ldots \ldots \ldots \ldots$

2.3.2 Updating regression coefficients $\beta \ldots \ldots \ldots \ldots$

2.3.3 Updating baseline hazard spline coefficients $\theta_{\lambda} \ldots \ldots \ldots$

2.3.4 Updating frailty density spline coefficients $\theta_{u} \ldots \ldots \ldots 6$

2.3.5 Updating baseline hazard parametric component parameters $\eta_{\lambda} \ldots \ldots \ldots \ldots \ldots \ldots$

2.3.6 Updating frailty density parametric component parameters $\eta_{u} \ldots \ldots \ldots \ldots \ldots \ldots \ldots \ldots$

2.3.7 Updating the weights $\phi_{\lambda}$ and $\phi_{u} \ldots \ldots \ldots \ldots$

2.3.8 Generate the error variance parameters $\sigma^{2} \ldots \ldots \ldots$. . 7

2.3.9 Setting Metropolis-Hastings tuning parameters . . . . . . 8

2.4 Reversible-Jump MCMC for adaptive knot selection . . . . . . . 8

2.4.1 Knot position change (move step) . . . . . . . . . . 9

2.4 .2 Knot addition (birth step) . . . . . . . . . . . . . . 9

2.4.3 Knot deletion (death step) . . . . . . . . . . . . 11

\footnotetext{
*Department of Operations Research \& Information Engineering

${ }^{\dagger}$ Department of Biological Statistics and Computational Biology

¥The Quality Institute and Department of Medicine

$\S$ Department of Medicine
} 
A Choice of parametric components . . . . . . . . . . . . . . . 12

A.1 Exponential baseline hazard . . . . . . . . . . . . . . . 12

A.2 Weibull baseline hazard . . . . . . . . . . . . . . 12

A.3 Gamma frailty density . . . . . . . . . . . . . . . . 13

A.4 Lognormal frailty density . . . . . . . . . . . . . . . 13

B Integrals involving B-splines . . . . . . . . . . . . . . . . . . . . 13

B.1 Cumulative hazard and normalization factor . . . . . . . . . 14

B.2 Moments of a normalized B-spline . . . . . . . . . . . 14

B.3 Construction of the penalty matrix on the integrated squared second derivative . . . . . . . . . . . . . . . . . . . . . . 14

C Gradients and Hessians . . . . . . . . . . . . . . . . . 15

References...................... . . . . . . . . . . . . . .

\section{Introduction}

This document contains supplementary materials for the article of the same title submitted for publication to the Electronic Journal of Statistics. Notation not defined in this document is defined as in the main paper. Section 2 provides additional details on the estimation procedure described in the main article; the appendices provide further information on a number of miscellaneous computational details not addressed in the main paper.

\section{Estimation procedure}

We introduce some additional but useful notation in Section 2.1; our choices for initializing the MCMC procedure may be found in Section 2.2. Detailed information on each of the Metropolis-Hastings parameter update steps is presented in Section 2.3, and the reversible-jump method used for knot selection is discussed in Section 2.4.

\subsection{Additional notation}

In the following, denote by $\boldsymbol{\beta}^{(h)}$ the estimated regression parameters at iteration $h$, with initial values corresponding to $h=0$, and analogously for the frailties and other estimated parameters $\boldsymbol{U}^{(h)}, \boldsymbol{\theta}_{\lambda}{ }^{(h)}, \boldsymbol{\theta}_{u}{ }^{(h)}, \boldsymbol{\eta}_{\lambda}^{(h)}, \boldsymbol{\eta}_{u}^{(h)}, \boldsymbol{\phi}^{(h)}, \boldsymbol{\sigma}^{2^{(h)}}, \boldsymbol{\tau}^{(h)}$. Also, denote the spline parameter weight vectors $\boldsymbol{w}_{\lambda}^{(h)}=e^{\boldsymbol{\theta}_{\lambda}^{(h)}}$ and $\boldsymbol{w}_{u}^{(h)}=$ $e^{\boldsymbol{\theta}_{u}^{(h)}} / \mathbf{1}_{m}^{T} e^{\boldsymbol{\theta}_{u}^{(h)}}$.

If adaptive knot selection is used, the number and placement of knots may also vary with each iteration. Denote the number of $\operatorname{knots} N_{\lambda}^{(h)}, N_{u}^{(h)}$ and corresponding spline parameter dimensions $K_{\lambda}^{(h)}, K_{u}^{(h)}$. Knots are located at $\boldsymbol{\xi}_{\lambda}^{(h)}, \boldsymbol{\xi}_{u}^{(h)}$, and correspond to B-spline bases $B_{\lambda k}^{(h)}(t)$ and $\tilde{B}_{u k}^{(h)}(x)$ for the hazard and frailty respectively. Without adaptive knot selection, $B_{\lambda k}^{(h)}(t)=B_{\lambda k}^{(0)}(t)$, with an analogous specification for the frailty splines. 
Define the basis function vector $\tilde{\boldsymbol{B}}_{u}^{(h)}(x)=\left(\tilde{B}_{u 1}^{(h)}(x), \ldots, \tilde{B}_{u K_{u}^{(h)}}^{(h)}(x)\right)$, with $\boldsymbol{B}_{\lambda}^{(h)}(t)$ specified in an analogous manner. Additionally, denote

$$
C_{\lambda k}^{(h)}(t)=\int_{0}^{t} B_{\lambda k}^{(h)}(s) d s, \quad \text { and } \quad E_{u k}^{(h)}=1-\int_{-\infty}^{\infty} x \tilde{B}_{u k}^{(h)}(x) d x,
$$

with corresponding vectors $\boldsymbol{C}_{\lambda}^{(h)}(t)$ and $\boldsymbol{E}_{u}^{(h)}$. The vector $\boldsymbol{E}_{u}^{(h)}$ is used in the course of parameter estimation to ensure that the frailty mean is 1 , for identifiability. Simple recursive formulas for computing these integrals may be found in appendix B.

In order to utilize full matrix notation, we define the following vectors and matrices:

$$
\begin{aligned}
& \tilde{\boldsymbol{U}}^{(h)}=\left[U_{11}^{(h)} \ldots U_{m J_{m}}^{(h)}\right]^{T} \quad \boldsymbol{\delta}=\left[\delta_{11} \ldots \delta_{m J_{m}}\right]^{T} \quad \boldsymbol{Z}=\left[\boldsymbol{Z}_{11} \ldots \boldsymbol{Z}_{m J_{m}}\right]^{T} \\
& \tilde{\boldsymbol{B}}_{u}^{(h)}=\left[\begin{array}{c}
\tilde{\boldsymbol{B}}_{u}\left(U_{1}^{(h)}\right) \\
\vdots \\
\tilde{\boldsymbol{B}}_{u}\left(U_{m}^{(h)}\right)
\end{array}\right] \quad \boldsymbol{B}_{\lambda}^{(h)}=\left[\begin{array}{c}
\boldsymbol{B}_{\lambda}^{(h)}\left(T_{11}\right) \\
\vdots \\
\boldsymbol{B}_{\lambda}^{(h)}\left(T_{m J_{m}}\right)
\end{array}\right] \quad \boldsymbol{C}_{\lambda}^{(h)}=\left[\begin{array}{c}
\boldsymbol{C}_{\lambda}^{(h)}\left(T_{11}\right) \\
\vdots \\
\boldsymbol{C}_{\lambda}^{(h)}\left(T_{m J_{m}}\right)
\end{array}\right] \\
& \boldsymbol{\lambda}_{p}^{(h)}=\left[\begin{array}{c}
\lambda_{p}\left(T_{11} \mid \boldsymbol{\eta}_{\lambda}^{(h)}\right) \\
\vdots \\
\lambda_{p}\left(T_{m J_{m}} \mid \boldsymbol{\eta}_{\lambda}^{(h)}\right)
\end{array}\right] \quad \boldsymbol{\Lambda}_{p}^{(h)}=\left[\begin{array}{c}
\Lambda_{p}\left(T_{11} \mid \boldsymbol{\eta}_{\lambda}^{(h)}\right) \\
\vdots \\
\Lambda_{p}\left(T_{m J_{m}} \mid \boldsymbol{\eta}_{\lambda}^{(h)}\right)
\end{array}\right] \quad \boldsymbol{f}_{p}^{(h)}=\left[\begin{array}{c}
f_{p}\left(U_{1} \mid \boldsymbol{\eta}_{u}^{(h)}\right) \\
\vdots \\
f_{p}\left(U_{m} \mid \boldsymbol{\eta}_{u}^{(h)}\right)
\end{array}\right] .
\end{aligned}
$$

Note that in $\tilde{\boldsymbol{U}}^{(h)}$, we use $U_{i j}^{(h)}=U_{i}^{(h)}$, giving a vector of length $\sum_{i} J_{i}$ (in constrast, $\boldsymbol{U}^{(h)}$ is of length $m$ ). The matrix $\boldsymbol{Z}$ has dimensions $\sum_{i} J_{i} \times p, \tilde{\boldsymbol{B}}_{u}^{(h)}$ has dimension $m \times K_{u}^{(h)}$, and matrices $\boldsymbol{B}_{\lambda}^{(h)}, \boldsymbol{C}_{\lambda}^{(h)}$ are $\sum J_{i} \times K_{\lambda}^{(h)}$. We will also denote by $D(\boldsymbol{x})$ a diagonal matrix with $\boldsymbol{x}$ on the diagonal.

At each iteration, this allows us to construct vectors containing the current baseline hazard, evaluated at each observed event time, and the current frailty density, evaluated at each of the current frailty values:

$$
\begin{aligned}
\boldsymbol{\lambda}_{0}^{(h)} & =\phi_{\lambda}^{(h)} \boldsymbol{B}_{\lambda}^{(h)} \boldsymbol{w}_{\lambda}^{(h)}+\left(1-\phi_{\lambda}^{(h)}\right) \boldsymbol{\lambda}_{0 p}^{(h)} \\
\boldsymbol{\Lambda}_{0}^{(h)} & =\phi_{\lambda}^{(h)} \boldsymbol{C}_{\lambda}^{(h)} \boldsymbol{w}_{\lambda}^{(h)}+\left(1-\phi_{\lambda}^{(h)}\right) \boldsymbol{\Lambda}_{0 p}^{(h)} \\
\boldsymbol{f}^{(h)} & =\phi_{u}^{(h)} \tilde{\boldsymbol{B}}_{u}^{(h)} \boldsymbol{w}_{u}^{(h)}+\left(1-\phi_{u}^{(h)}\right) \boldsymbol{f}_{p}^{(h)},
\end{aligned}
$$

The dependence of these quantities on other parameters will be used implicitly in the construction of conditional likelihoods for the Metropolis-Hastings steps.

Lastly, define tuning parameters $\gamma_{\nu}, \gamma_{\lambda}, \gamma_{\beta}, \gamma_{u}$ chosen to make acceptance probabilities in the Metropolis-Hastings algorithm close to $25 \%$. These may be set manually or can be selected by an adaptive procedure during the burn-in phase of the chain. We present a simplistic but effective adaptive procedure to choose MCMC tuning parameters during the burn-in phase in Section 2.3.9. 


\subsection{Obtaining initial values}

In principle, the Metropolis-Hastings chain can be initialized at any value. However, we find that good starting values hasten the convergence of the chain, and reduce the risk of numerical problems. This section contains initial values that we have found to yield good results. They are in part found by using computationally inexpensive frequentist methods, and by maximizing conditional likelihoods.

For the B-spline $\boldsymbol{B}_{\lambda}(t)$ specifying the baseline hazard, with events occurring at times $T_{i j}$, we set the initial number of interior knots to

$$
N_{\lambda}^{(0)}=\min \left(\frac{\sum_{i} J_{i}}{4}, 35\right),
$$

distributed evenly or by quantiles along the range of observed event times. In addition, if the spline order $Q_{\lambda}>1$, we define repeated exterior knots located at the boundaries in order to ensure that $\boldsymbol{B}_{\lambda}^{(h)}(t)$ is supported exactly on the range of event times.

Analogously, for the (unnormalized) B-spline $\boldsymbol{B}_{u}^{(h)}(x)$ of order $Q_{u}$ defining the frailty density, we choose a support range $\left(U_{\min }, U_{\max }\right)$, and distribute $N_{u}^{(0)}=$ $\min (m / 4,35)$ knots evenly across the range. The range may be chosen a priori, or it may be allowed to depend on the initial estimates of the frailties from a parametric frailty model.

We set the initial values for all parameters as follows:

- Hyperparameters $\boldsymbol{\alpha}=\left(\boldsymbol{\alpha}_{\beta}, \boldsymbol{\alpha}_{\lambda}, \boldsymbol{\alpha}_{u}\right)$ are fixed as

$$
\alpha_{\beta 1}=\alpha_{\beta 2}=\alpha_{\lambda 1}=\alpha_{\lambda 2}=\alpha_{u 1}=\alpha_{u 2}=0.01
$$

indicating diffuse priors on the regression and spline parameters. Hyperparameters $\boldsymbol{\alpha}_{\eta}$ may depend on the parametric form chosen, but for the parameterizations given in Section A, analogous diffuse Gaussian priors are appropriate. The hyperparameters $\boldsymbol{\alpha}_{\phi_{\lambda}}, \boldsymbol{\alpha}_{\phi_{u}}$ determine the form of the Beta prior on the weights $\phi_{\lambda}, \phi_{u}$.

- Initial values for the frailties $\boldsymbol{U}^{(0)}$ and for the parameters $\boldsymbol{\beta}^{(0)}$ can be obtained by fitting a frequentist proportional hazards frailty model to the data (e.g. via coxph in $\mathrm{R}$ ). The frailty distribution should match the parametric component, if available.

- Parametric baseline hazard parameters $\boldsymbol{\eta}_{\lambda}$ can be initialized by fitting a parametric proportional hazards model to the data (e.g. via survreg in R). Initial values for the parametric frailty density parameters $\boldsymbol{\eta}_{u}$ can be obtained from the frailty model fit previously.

- Initial values for the variance parameters $\boldsymbol{\sigma}^{2^{(0)}}, \boldsymbol{\tau}^{(0)}$ are set as

$$
\sigma_{\beta}^{2(0)}=\sigma_{\lambda}^{2(0)}=\sigma_{u}^{2^{(0)}}=\tau_{\lambda}^{(0)}=\tau_{u}^{(0)}=0.1
$$


- Initial values for the spline coefficients $\boldsymbol{\theta}_{\lambda}{ }^{(0)}, \boldsymbol{\theta}_{u}{ }^{(0)}$ can be found by maximizing the conditional log-likelihoods $\ell\left(\boldsymbol{\theta}_{\lambda} \mid \boldsymbol{U}^{(0)}, \boldsymbol{\beta}^{(0)}, \boldsymbol{\eta}^{(0)}, \boldsymbol{\phi}^{(0)}, \boldsymbol{\sigma}^{2(0)}\right)$ and $\ell\left(\boldsymbol{\theta}_{u} \mid \boldsymbol{U}^{(0)}, \boldsymbol{\beta}^{(0)}, \boldsymbol{\eta}^{(0)}, \phi^{(0)}, \boldsymbol{\sigma}^{2(0)}\right.$ ) (see Section 2.3.3 and Section 2.3.4 for the likelihoods. Formulas for the gradients are given in appendix $\mathrm{C}$.

For many (but not all) of the parameters above, the intuition behind the proposed choice is to try and begin sampling somewhere close to the relevant posterior mode.

\subsection{Metropolis-Hastings MCMC steps}

Once initial values have been obtained, the algorithm begins running the MCMC sampling loop for a specified number of iterations. The first stage consists of successive Gibbs sampling steps, in which, given iteration $h$ parameter estimates, each set of parameters is updated in turn by Metropolis-Hastings. The following subsections detail the likelihoods for each of these parameters conditional on the remainder, which for brevity are denoted by an ellipsis.

\subsubsection{Updating the frailties $U_{i}$}

The loglikelihood for the frailty parameters $\boldsymbol{U}$ conditional on the remaining parameters is given by portions of the full likelihood, that is:

$$
\ell(\boldsymbol{U} \mid \ldots)=\sum_{i=1}^{m}\left[\sum_{j=1}^{J_{i}}\left(\delta_{i j} \log U_{i}-U_{i} \boldsymbol{\Lambda}_{0}^{(h)} e^{\boldsymbol{Z}_{i j}^{T} \boldsymbol{\beta}^{(h)}}\right)+\log f_{i}^{(h)}\right] .
$$

Conditional on the remaining parameters, the frailties $U_{i}$ are independent. We thus generate candidates individually for the $h+1$-st iteration from a Gamma transition kernel with mean $U_{i}^{(h)}$ and variance $\gamma_{\nu}$, that is,

$$
\tilde{U}_{i}^{(h+1)} \sim \operatorname{Gamma}\left\{\gamma_{\nu}^{-1}\left(U_{i}^{(h)}\right)^{2}, \gamma_{\nu}\left(U_{i}^{(h)}\right)^{-1}\right\}
$$

where $\gamma_{\nu}$ is a tuning parameter. Each candidate is accepted with probability

$$
\rho_{u}=\min \left\{1, \frac{\mathcal{L}\left(\tilde{U}_{i}^{(h+1)} \mid \ldots\right) \mathcal{T}\left(\tilde{U}_{i}^{(h+1)}, U_{i}^{(h)}\right)}{\mathcal{L}\left(U_{i}^{(h)} \mid \ldots\right) \mathcal{T}\left(U_{i}^{(h)}, \tilde{U}_{i}^{(h+1)}\right)}\right\}
$$

where $\mathcal{L}$ is the posterior likelihood, $\mathcal{T}\left(x, x^{\prime}\right)$ is a gamma transition kernel with mean $x$ and variance $\gamma_{\nu}$ evaluated at $x^{\prime}$.

\subsubsection{Updating regression coefficients $\beta$}

The loglikelihood for the regression coefficients conditional on the remaining parameters can be written as

$$
\ell(\boldsymbol{\beta} \mid \ldots)=\boldsymbol{\delta}^{T} \boldsymbol{Z} \boldsymbol{\beta}-\tilde{\boldsymbol{U}}^{(h)^{T}} D\left(\boldsymbol{\Lambda}_{0}^{(h)}\right) e^{\boldsymbol{Z} \boldsymbol{\beta}}-\frac{\boldsymbol{\beta}^{T} \boldsymbol{\beta}}{2 \sigma_{\beta}^{2}} .
$$


Candidates for the $h+1$-st iteration can be generated from $N\left(\boldsymbol{\beta}^{(h)}, \gamma_{\beta} \boldsymbol{\Sigma}_{\beta}\right)$, where $\boldsymbol{\Sigma}_{\beta}$ is the inverse Hessian of the likelihood in eq. (2.2) evaluated at the initial values (see eq. (C.2)).

\subsubsection{Updating baseline hazard spline coefficients $\theta_{\lambda}$}

The nonparametric estimate of the baseline hazard depends on parameters $\boldsymbol{\theta}_{\lambda}$. The loglikelihood for these coefficients conditional on the remaining parameters is given by

$$
\ell\left(\boldsymbol{\theta}_{\lambda} \mid \ldots\right)=\boldsymbol{\delta}^{T} \log \boldsymbol{\lambda}_{0}^{(h)}-\tilde{\boldsymbol{U}}^{(h)^{T}} D\left(\boldsymbol{\Lambda}_{0}^{(h)}\right) e^{\boldsymbol{Z} \boldsymbol{\beta}^{(h)}}-\frac{p_{\lambda}\left(\boldsymbol{\theta}_{\lambda}\right)}{2 \sigma_{\lambda}^{2}} .
$$

Candidates for the $h+1$-st iteration can be generated one by one from $N\left(\theta_{\lambda k}^{(h)}, \gamma_{\lambda}\right)$, where $\gamma_{\lambda}$ is a tuning parameter.

\subsubsection{Updating frailty density spline coefficients $\theta_{u}$}

The loglikelihood for the frailty density spline coefficients $\boldsymbol{\theta}_{u}$ conditional on the remaining parameters is given by

$$
\ell\left(\boldsymbol{\theta}_{u} \mid \boldsymbol{U}, \boldsymbol{\beta}, \boldsymbol{\theta}_{\lambda}\right)=\mathbf{1}_{m}^{T} \log \boldsymbol{f}^{(h)}-\frac{p_{u}\left(\boldsymbol{\theta}_{u}\right)}{2 \sigma_{u}^{2}} .
$$

In generating candidates for the $h+1$-st iteration, it is important for identifiability to ensure that the mean of the frailty density is fixed at 1 . This condition can be expressed as the constraint

$$
\boldsymbol{E}_{u}^{(h)} e^{\boldsymbol{\theta}_{u}^{(h)}}=0 .
$$

where $\boldsymbol{E}_{u}^{(h)}$ is defined in eq. (2.1). We therefore generate candidates in pairs, in such a way that the constraint is always satisfied: for each $k$, we first generate a candidate $\tilde{\theta}_{u k}^{(h+1)} \sim N\left(\boldsymbol{\theta}_{u k}^{(h)}, \gamma_{u}\right)$, where $\gamma_{u}$ is a tuning parameter. We then choose a random index $m$ among the remainder, and adjust the candidate $\tilde{\theta}_{u m}^{(h+1)}$ as

$$
\tilde{\theta}_{u m}^{(h+1)}=\log \left\{\frac{1}{E_{u m}^{(h)}}\left(E_{u k}^{(h)}\left(e^{\theta_{u k}^{(h)}}-e^{\tilde{\theta}_{u k}^{(h+1)}}\right)+E_{u m}^{(h)} e^{\theta_{u m}^{(h)}}\right)\right\},
$$

which ensures that the pair $\left(\theta_{u k}^{(h+1)}, \theta_{u m}^{(h+1)}\right)$ continues to satisfy the constraint. Since this mode of candidate generation still has a symmetric transition kernel, each pair is accepted with a standard Metropolis probability. 


\subsubsection{Updating baseline hazard parametric component parameters $\eta_{\lambda}$}

The parametric component $\lambda_{0 p}$ of the baseline hazard $\lambda_{0}$ depends on parameters $\boldsymbol{\eta}_{\lambda}$. The loglikelihood for these parameters is given by

$$
\ell\left(\boldsymbol{\eta}_{\lambda} \mid \ldots\right)=\boldsymbol{\delta}^{T} \log \boldsymbol{\lambda}_{0}^{(h)}-\tilde{\boldsymbol{U}}^{(h)^{T}} D\left(\boldsymbol{\Lambda}_{0}^{(h)}\right) e^{\boldsymbol{Z} \boldsymbol{\beta}^{(h)}}+\pi_{\lambda}\left(\boldsymbol{\eta}_{\lambda} \mid \tau_{\lambda}^{(h)}\right) .
$$

Candidate generation may depend on the parametric form, but we have found that if the distributions are parametrized in such a way that their parameters are unconstrained, multivariate Gaussian transition kernels yield good results. Some effective parametrizations are discussed in appendix A.

\subsubsection{Updating frailty density parametric component parameters $\eta_{u}$}

The loglikelihood for the parameters corresponding to the parametric component of the baseline hazard is given by

$$
\ell\left(\boldsymbol{\eta}_{u} \mid \ldots\right)=\mathbf{1}_{m}^{T} \log \boldsymbol{f}^{(h)}+\pi_{u}\left(\boldsymbol{\eta}_{u} \mid \tau_{u}^{(h)}\right) .
$$

Again, candidate generation is discussed in appendix A.

\subsubsection{Updating the weights $\phi_{\lambda}$ and $\phi_{u}$}

The relative weights of the parametric and nonparametric components for the baseline hazard and frailty curves have the following likelihoods:

$$
\begin{aligned}
\ell\left(\phi_{\lambda} \mid \ldots\right)= & \boldsymbol{\delta}^{T} \log \boldsymbol{\lambda}_{0}^{(h)}-\tilde{\boldsymbol{U}}^{(h)^{T}} D\left(\boldsymbol{\Lambda}_{0}^{(h)}\right) e^{\boldsymbol{Z} \boldsymbol{\beta}^{(h)}} \\
& +\left(\alpha_{\phi_{\lambda} 1}-1\right) \log \phi_{\lambda}^{(h)}+\left(\alpha_{\phi_{\lambda} 2}-1\right) \log \left(1-\phi_{\lambda}^{(h)}\right) \\
\ell\left(\phi_{u} \mid \ldots\right)= & \mathbf{1}_{m}^{T} \log \boldsymbol{f}^{(h)}+\left(\alpha_{\phi_{u} 1}-1\right) \log \phi_{u}^{(h)}+\left(\alpha_{\phi_{u} 2}-1\right) \log \left(1-\phi_{u}^{(h)}\right)
\end{aligned}
$$

We generate candidates for $\tilde{\phi}_{\lambda}^{(h+1)}$ using a Beta transition kernel with mean $\phi_{\lambda}^{(h)}$ and variance $\gamma_{\phi \lambda}$, where the latter is a tuning parameter, and analogously for $\tilde{\phi}_{u}^{(h+1)}$.

\subsubsection{Generate the error variance parameters $\sigma^{2}$}

Parameters $\left(\sigma_{\beta}^{2}, \sigma_{\lambda}^{2}, \sigma_{u}^{2}\right)$ are sampled from the following inverse-gamma distributions:

$$
\begin{aligned}
\sigma_{\beta}^{2} & \sim I G\left(\frac{p}{2}+\alpha_{\beta 1}, \frac{\boldsymbol{\beta}^{(h)^{T}} \boldsymbol{\beta}^{(h)}}{2}+\alpha_{\beta 2}\right) \\
\sigma_{\lambda}^{2} & \sim I G\left(\frac{K_{\lambda}^{(h)}}{2}+\alpha_{\lambda 1}, \frac{p_{\lambda}\left(\boldsymbol{\theta}_{\lambda}^{(h)}\right)}{2}+\alpha_{\lambda 2}\right) \\
\sigma_{u}^{2} & \sim I G\left(\frac{K_{u}^{(h)}}{2}+\alpha_{u 1}, \frac{p_{u}\left(\boldsymbol{\theta}_{u}^{(h)}\right)}{2}+\alpha_{u 2}\right)
\end{aligned}
$$


For the remaining parameters corresponding to the parametric components, other priors may be appropriate depending on the parametric form and parametrization chosen. We discuss these in appendix A.

\subsubsection{Setting Metropolis-Hastings tuning parameters}

The preceding steps in Section 2.3.1-2.3.8 depend on tuning parameters $\gamma_{\nu}, \gamma_{\beta}$, $\gamma_{\lambda}, \gamma_{u}, \gamma_{\eta \lambda}, \gamma_{\eta_{u}}, \gamma_{\phi \lambda}, \gamma_{\phi_{u}}$, which must be set in such a way that the acceptance rate of each of the Metropolis-Hastings steps is approximately 25\%. It is infeasible to calibrate so many parameters by hand, so we offer the following heuristic:

During the burn-in phase of length $B$ iterations, the MCMC loop of Section 2.3.1-2.3.8 and possibly Section 2.4 may be interrupted every $b$ iterations, $b<B$. The tuning parameters and acceptance rates used during each previous interval of length $b$ can then be used to predict the values of the tuning parameters for which the acceptance rates are $25 \%$, e.g. using linear regression, and the results can be used as tuning parameter values for the next $b$ iterations. The value of $b$ should be chosen so that $B / b$ is sufficient to yield a large number of evaluations. After the end of the burn-in, the tuning parameters are held fixed.

Although simplistic, we have found that this method works well, and yields acceptance rates that are very close to $25 \%$.

\subsection{Reversible-Jump MCMC for adaptive knot selection}

In all the steps discussed in Section 2.3, the number of knots in the model, and hence the dimension of the spline parameters $\boldsymbol{\theta}_{\lambda}, \boldsymbol{\theta}_{u}$, has remained fixed. In order to enable adaptive knot selection, we not only allow knots to move, but also permit changes in dimension, such as adding a knot (birth step) or deleting a knot (death step).

We discuss the procedure in general terms only, since it is identical for the hazard spline and the frailty density spline, and we omit subscripts that identify the parameters as referring to either curve. As before, let $N^{(h)}$ denote the number of interior spline knots $\boldsymbol{\xi}^{(h)}$, and $\boldsymbol{\theta}^{(h)}$ the spline parameter vector of length $K^{(h)}=N^{(h)}+Q$, at iteration $h$. Let $\pi_{N}(n)$ denote the prior on the number of knots.

As detailed in Green (1995), changes in model dimension in reversible-jump MCMC are subject to a "dimension-matching" constraint. Typically, transitions between a model indexed by a parameter set $\boldsymbol{\theta}$ of dimension $k$ and a candidate model indexed by parameters $\tilde{\boldsymbol{\theta}}$ of dimension $\tilde{k}$ are accomplished by generating $m$ uniform random numbers $\boldsymbol{u}$ and computing the candidate by a deterministic function $\tilde{\boldsymbol{\theta}}=\tilde{\boldsymbol{\theta}}(\boldsymbol{\theta}, \boldsymbol{u})$. For the reverse move, one generates $\tilde{m}$ random numbers $\tilde{\boldsymbol{u}}$ and computes the candidate as $\boldsymbol{\theta}=\boldsymbol{\theta}(\tilde{\boldsymbol{\theta}}, \tilde{\boldsymbol{u}})$. To ensure reversibility, the mapping between $(\boldsymbol{\theta}, \boldsymbol{u})$ and $(\tilde{\boldsymbol{\theta}}, \tilde{\boldsymbol{u}})$ must be bijective, and in particular, the dimensionmatching constraint $m+k=\tilde{m}+\tilde{k}$ must hold.

In our context, adaptive knot selection requires three types of steps: the "move" step, in which the position of a single knot is changed to some new 
point between its neighbor knots, the "birth" step, in which a new knot is added after a randomly chosen knot and the dimension of the spline parameter $\boldsymbol{\theta}$ increases, and the "death" step, in which a randomly chosen knot is removed and the dimension of the parameter decreases. The move step requires no dimension change, and Metropolis-Hastings methods are sufficient. The death and birth steps however are subject to the reversibility and dimension-matching constraints.

Following Denison, Mallick and Smith (1998), at each iteration we choose randomly whether to execute a birth, death, or move step. Given $N^{(h)}=n$, the probabilites $b_{n}, d_{n}, m_{n}$ of birth, death and move steps respectively are set to:

$b_{n}=c \min \left\{1, \frac{\pi_{N}(n+1)}{\pi_{N}(n)}\right\}, \quad d_{n}=c \min \left\{1, \frac{\pi_{N}(n-1)}{\pi_{N}(n)}\right\}, \quad m_{n}=1-b_{n}-d_{n}$,

where the constant $c$ controls the rate of dimension-changing steps, and is set to $c=0.4$ as in Denison, Mallick and Smith (1998). These parameters are chosen so that

$$
b_{n} \pi_{N}(n)=d_{n+1} \pi_{N}(n+1) .
$$

We give details on the move step in Section 2.4.1, the birth step in Section 2.4.2, and the death step in Section 2.4.3.

\subsubsection{Knot position change (move step)}

In the move step, a single knot position $\xi_{k}^{(h)}$ to be moved is chosen uniformly from the set of interior knots, and changed to a random new candidate position located between its neighboring knots. That is, the candidate knot position $\tilde{\xi}_{k}^{(h)}$ is selected uniformly from the set of candidate locations $\xi^{c} \in \boldsymbol{\xi}^{c}$ such that $\xi_{k-1}^{(h)}<\xi^{c}<\xi_{k+1}^{(h)}$. The spline parameters $\boldsymbol{\theta}$ remain unchanged.

Since the prior on the knot positions is discrete uniform over the set of candidate knots, the prior probabilities for knots $\boldsymbol{\xi}^{(h)}$ and the candidate $\tilde{\boldsymbol{\xi}}^{(h+1)}$ are identical. Since no dimension change is required, the new knot positions are accepted with probability

$$
\rho=\min \left\{1, \frac{\mathcal{L}\left(\boldsymbol{\theta} \mid \tilde{\boldsymbol{\xi}}^{(h+1)}, \ldots\right)}{\mathcal{L}\left(\boldsymbol{\theta} \mid \boldsymbol{\xi}^{(h)}, \ldots\right)}\right\},
$$

where $\mathcal{L}$ is posterior spline parameter likelihood given in eq. (2.3) or eq. (2.4) for the hazard and frailty spline respectively. Note that these likelihoods depend on the knot positions through $\boldsymbol{\lambda}_{0}^{(h)}$ and $\boldsymbol{f}^{(h)}$ for the hazard and frailty respectively.

\subsubsection{Knot addition (birth step)}

In the birth move, a random unoccupied candidate knot $\xi^{c} \in \boldsymbol{\xi}^{c}$ is chosen to be added to the current set of knots $\boldsymbol{\xi}^{(h)}$, of length $N^{(h)}$. Denote by $k$ the interval of 
the current knot set containing $\xi^{c}$, so that $\xi_{k}^{(h)}<\xi^{c}<\xi_{k+1}^{(h)}$. The new candidate knot set is then given by

$$
\tilde{\boldsymbol{\xi}}^{(h+1)}=\left\{\xi_{1}^{(h)}, \ldots, \xi_{k}^{(h)}, \xi^{c}, \xi_{k+1}^{(h)}, \ldots, \xi_{N^{(h)}}^{(h)}\right\}
$$

of length $\tilde{N}^{(h+1)}=N^{(h)}+1$.

The set of spline coefficients $\boldsymbol{\theta}^{(h)}$ of length $K^{(h)}$ must be updated to a candidate set $\tilde{\boldsymbol{\theta}}^{(h+1)}$ of length $\tilde{K}^{(h+1)}=K^{(h)}+1$. There are simple rules for nondestructively inserting a new knot into a B-spline function (de Boor, 2001), but using these directly would violate the reversibility and dimension-matching constraint between the birth and death moves mentioned earlier. Since the birth move begins in a model of dimension $K^{(h)}$ and its reverse begins at dimension $K^{(h)}+1$, we need to generate an additional random number for the birth move. Intuitively, since removing a knot is a destructive procedure and may cause the shape of the curve to change, we must during the birth move be able to generate the set of curves that would reduce to the original curve upon removal of the new knot.

To do this, we compute the candidate spline parameters $\tilde{\boldsymbol{\theta}}^{(h+1)}$ for inserting a knot $\xi^{c} \in\left(\xi_{k}^{(h)}, \xi_{k+1}^{(h)}\right)$ as follows:

$$
\tilde{\theta}_{j}^{(h+1)}= \begin{cases}\theta_{j}^{(h)} & \text { if } j \leq k+1 \\ \theta_{j-1}^{(h)} & \text { if } j>k+Q \\ \log \left(r_{j} e^{\theta_{j}^{(h)}}+\left(1-r_{j}\right) e^{\theta_{j-1}^{(h)}}\right) & \text { if } k+1<j<k+Q \\ \log \left(u e^{\theta_{j}^{(h)}}+(1-u) e^{\theta_{j-1}^{(h)}}\right) & \text { if } j=k+Q\end{cases}
$$

where $r_{j}=\left(\xi^{c}-\xi_{j-Q}^{(h)}\right) /\left(\xi_{j-1}^{(h)}-\xi_{j-Q}^{(h)}\right)$, and $u \sim U(0,1)$. These rules correspond to the deterministic rules in de Boor (2001), except that the parameter $\tilde{\theta}_{k+Q}^{(h+1)}$ is perturbed by a random amount, rather than by the knot ratio $r_{k+Q}$.

The prior ratio for the birth move is given by product of the ratio of the priors on the number of knots, the priors on the knot positions, and the priors on the spline parameters:

$$
R_{P}=\frac{\pi_{N}\left(N^{(h)}+1\right)}{\pi_{N}\left(N^{(h)}\right)} \cdot \frac{N^{(h)}+1}{M-N^{(h)}} \cdot \frac{\pi\left(\tilde{\boldsymbol{\theta}}^{(h+1)} \mid \sigma^{2}\right)}{\pi\left(\boldsymbol{\theta}^{(h)} \mid \sigma^{2}\right)},
$$

since the prior on the knot positions is that they are randomly chosen among the candidate knots, so that $\pi\left(\boldsymbol{\xi}^{(h)}\right)=\left[\left(\begin{array}{c}M \\ N^{(h)}\end{array}\right)\right]^{-1}$. The transition ratio is given by

$$
R_{T}=\frac{d_{N^{(h)}+1} /\left(N^{(h)}+1\right)}{b_{N^{(h)}} /\left(M-N^{(h)}\right)} .
$$

Note that together with eq. (2.5), this implies that

$$
R_{P} \cdot R_{T}=\frac{\pi\left(\tilde{\boldsymbol{\theta}}^{(h+1)} \mid \sigma^{2}\right)}{\pi\left(\boldsymbol{\theta}^{(h)} \mid \sigma^{2}\right)}=(2 \pi \sigma)^{-\frac{1}{2}} \exp \left(\frac{p\left(\tilde{\boldsymbol{\theta}}^{(h+1)}\right)-p\left(\boldsymbol{\theta}^{(h)}\right)}{2 \sigma^{2}}\right) .
$$


The likelihood ratio $R_{L}$ is given by the ratios of the likelihoods for the spline parameters $\boldsymbol{\theta}^{(h)}$, whose logarithms are given by either eq. (2.3) or eq. (2.4), without the prior penalty terms. Lastly, the Jacobian for the transformation from $\left(\boldsymbol{\theta}^{(h)}, u\right)$ to $\left(\tilde{\boldsymbol{\theta}}^{(h+1)}\right)$ in eq. $(2.6)$ is

$$
|J|=\left|\frac{\left(\exp \left(\theta_{k+Q}^{(h)}\right)-\exp \left(\theta_{k+Q-1}^{(h)}\right)\right)}{\exp \left(\tilde{\theta}_{k+Q}^{(h+1)}\right)} \prod_{j=k+2}^{k+Q-1} \frac{r_{j} \cdot \exp \left(\theta_{j}^{(h)}\right)}{\exp \left(\tilde{\theta}_{j}^{(h+1)}\right)}\right|
$$

The candidate number of knots $\tilde{N}^{(h+1)}$ and spline parameters $\tilde{\boldsymbol{\theta}}^{(h+1)}$ are then accepted with probability

$$
\rho=\min \left\{1, R_{L} \cdot R_{P} \cdot R_{T} \cdot|J|\right\} .
$$

\subsubsection{Knot deletion (death step)}

In the death step, a single knot $\xi_{k}^{(h)}$ is chosen uniformly from the set of knots $\boldsymbol{\xi}^{(h)}$ to be removed. The candidate knot set for the next iteration is then $\tilde{\boldsymbol{\xi}}^{(h+1)}=\left\{\xi_{1}^{(h)}, \ldots, \xi_{k-1}^{(h)}, \xi_{k+1}^{(h)}, \ldots, \xi_{N^{(h)}}^{(h)}\right\}$. The spline parameters are correspondingly adjusted by the inverse of the transformation in eq. (2.6), that is, by deleting the parameter $\theta_{k+Q-1}^{(h)}$ and adjusting the remaining parameters as

$$
\tilde{\theta}_{j}^{(h+1)}= \begin{cases}\theta_{j}^{(h)} & \text { if } j<k+1 \\ \theta_{j+1}^{(h)} & \text { if } j \geq k+Q-1 \\ \log \left(\frac{1}{r_{j}} e^{\theta_{j}^{(h)}}-\frac{1-r_{j}}{r_{j}} e^{\theta_{j-1}^{(h)}}\right) & \text { if } k+1 \leq j<k+Q-1\end{cases}
$$

Because the birth and death moves are symmetrically defined, the likelihood ratio, prior ratio, transition ratio and Jacobian determinant are the inverses of those in eq. (2.7). 


\section{Appendix A: Choice of parametric components}

Both the baseline hazard and frailty density may have optional parametric components. In this section, we present some of the possible choices of distributions, along with appropriate priors, initial values and estimation procedures.

\section{A.1. Exponential baseline hazard}

The exponential baseline hazard can be parametrized by a constant log-hazard $\boldsymbol{\eta}_{\lambda}=\eta_{\lambda}$, so that the hazard function is

$$
\lambda_{0 p}\left(t, \boldsymbol{\eta}_{\lambda}\right)=\exp \left(\eta_{\lambda}\right), \quad \Lambda_{0 p}\left(t, \boldsymbol{\eta}_{\lambda}\right)=t \exp \left(\eta_{\lambda}\right) .
$$

A reasonable prior for $\eta_{\lambda}$ is Gaussian with variance $\tau_{\lambda}$ :

$$
\log \pi_{\lambda}\left(\eta_{\lambda} \mid \tau_{\lambda}\right)=-\frac{1}{2} \log \sigma_{\lambda}^{2}-\frac{\eta_{\lambda}^{2}}{2 \tau_{\lambda}}
$$

with an inverse-Gamma prior for $\tau_{\lambda}$ :

$$
\log \pi_{\tau_{\lambda}}\left(\tau_{\lambda} \mid \boldsymbol{\alpha}_{\sigma_{\lambda}^{2}}\right)=-\left(\alpha_{\tau_{\lambda} 1}+1\right) \log \tau_{\lambda}-\frac{\alpha_{\tau_{\lambda} 2}}{\tau_{\lambda}}
$$

depending on hyperparameters $\alpha_{\tau_{\lambda} 1}, \alpha_{\tau_{\lambda} 2}$ fixed at 0.01 .

Candidates for the $k+1$-st iteration $\eta_{\lambda}$ may be generated as $N\left(\eta_{\lambda}^{(h)}, \gamma_{\eta_{\lambda}}\right)$, where $\gamma_{\eta_{\lambda}}$ is a tuning parameter chosen to make the acceptance probability close to $25 \%$.

\section{A.2. Weibull baseline hazard}

The Weibull baseline hazard is parametrized by a $\log$-hazard $\eta_{\lambda 1}$ and $\log$ scale parameter $\eta_{\lambda 2}$, so the hazard function is

$\lambda_{0 p}\left(t, \boldsymbol{\eta}_{\lambda}\right)=\exp \left(\eta_{\lambda 1}+\eta_{\lambda 2}+\left(e^{\eta_{\lambda 2}}-1\right) \log t\right), \quad \Lambda_{0 p}\left(t, \boldsymbol{\eta}_{\lambda}\right)=\exp \left(\eta_{\lambda 1}\right) t^{\exp \left(\eta_{\lambda 2}\right)}$

Similar to the exponential case, assume the priors for $\boldsymbol{\eta}_{\lambda}$ are independent Gaussian with variances $\boldsymbol{\tau}_{\lambda}=\left(\tau_{\lambda 1}, \tau_{\lambda 2}\right)$ :

$$
\log \pi_{\lambda}\left(\eta_{\lambda i} \mid \boldsymbol{\tau}_{\lambda}\right)=-\sum_{i} \log \tau_{\lambda i}-\frac{\eta_{\lambda i}^{2}}{2 \tau_{\lambda_{i}}}
$$

with inverse-Gamma priors for $\tau_{\lambda i}$ :

$$
\log \pi_{\tau \lambda i}\left(\tau_{\lambda i} \mid \boldsymbol{\alpha}_{\tau_{\lambda} i}\right)=-\left(\alpha_{\tau_{\lambda} i 1}+1\right) \log \tau_{\lambda}-\frac{\alpha_{\tau_{\lambda} i 2}}{\tau_{\lambda}}
$$

depending on hyperparameters $\alpha_{\tau_{\lambda} i 1}, \alpha_{\tau_{\lambda} i 2}$ fixed at 0.01 .

Candidates for the $k+1$-st iteration $\boldsymbol{\eta}_{\lambda}$ may be generated independently as $N\left(\eta_{\lambda i}^{(h)}, \gamma_{\eta_{\lambda} i}\right)$, where $\gamma_{\eta_{\lambda} i}$ are tuning parameters chosen to make the acceptance probability close to $25 \%$. It is possible to simplify the prior structure somewhat by assuming that $\tau_{\lambda 1}=\tau_{\lambda 2}=\tau_{\lambda}$, and we have found this to be equally effective. 


\section{A.3. Gamma frailty density}

The gamma frailty distribution parametrized by its log-variance $\eta_{u}$ results in the following parametric density component:

$$
f_{p}\left(x, \eta_{u}\right)=x^{\exp \left(-\eta_{u}\right)-1} \frac{\exp \left(-\eta_{u}\right)^{\exp \left(-\eta_{u}\right)} e^{-\exp \left(-\eta_{u}\right) x}}{\Gamma\left(\exp \left(-\eta_{u}\right)\right)}
$$

Similar to the Exponential baseline hazard case case, let the the prior for $\eta_{u}$ be univariate Gaussian with variance $\tau_{u}$, in which case the hierarchical structure and candidate generation is identical.

\section{A.4. Lognormal frailty density}

The lognormal frailty density parametrized by a log-variance parameter $\eta_{u}$ results in the following parametric density component:

$$
f_{p}\left(x, \eta_{u}\right)=\frac{\exp \left(-\frac{\left(\log x+\frac{1}{2} \exp \left(\eta_{u}\right)\right)^{2}}{2 \exp \left(\eta_{u}\right)}\right)}{x \sqrt{2 \pi \exp \left(\eta_{u}\right)}}
$$

This is a lognormal distribution with mean 1 and variance $e^{\exp \left(\eta_{u}\right)}-1$. Choosing a Gaussian prior for the log-variance allows a parametrization identical to the Gamma case above.

\section{Appendix B: Integrals involving B-splines}

In several places we require quantities requiring the computation of an integral that depends on one or more B-splines. Consider first the B-spline $\boldsymbol{B}_{\lambda}(t)$ specifying the baseline hazard. Recall that events occur at times $T_{i j}$, and the number of interior knots is set to

$$
N_{\lambda}=\min \left(\frac{\sum_{i} J_{i}}{4}, 35\right)
$$

and the knots will be positioned at $\xi_{\lambda k}=\min \left(T_{i j}\right)+k \Delta_{\lambda}$, for $k=0, \ldots, N_{\lambda}+1$, where

$$
\Delta_{\lambda}=\frac{\max \left(T_{i j}\right)-\min \left(T_{i j}\right)}{N_{\lambda}+1} .
$$

In addition, if the spline order $Q_{\lambda}>1$, define repeated exterior knots located at the boundaries, so that $\xi_{\lambda(-1)}, \ldots, \xi_{\lambda\left(-Q_{\lambda}+1\right)}=\xi_{\lambda 0}$ and $\xi_{\lambda\left(N_{\lambda}+1\right)}, \ldots, \xi_{\lambda\left(N_{\lambda}+Q_{\lambda}\right)}=$ $\xi_{\lambda N_{\lambda}}$. Note that $B_{\lambda k}$ is supported on the range $\left(\xi_{\lambda\left(k-Q_{\lambda}\right)}, \xi_{\lambda k}\right)$.

For the (unnormalized) B-spline $\boldsymbol{B}_{u}(x)$ of order $Q_{u}$ defining the frailty density, the number and placement of knots $\xi_{u k}$ are determined analogously.

In this section we give formulas for the integrals $C_{\lambda}\left(T_{i j}\right)$ (the cumulative baseline hazard), $E_{u k}$ (the mean of each normalized spline component), the normalization factor to produce normalized B-splines $\tilde{\boldsymbol{B}}_{u}(x)$, and the integrals for a penalty over the second derivative. 


\section{B.1. Cumulative hazard and normalization factor}

A formula for the indefinite integral of a B-spline is given in Cox (1982), allowing us to compute

$C_{\lambda k}(x)=\int_{0}^{x} B_{\lambda k}(t) d t= \begin{cases}\frac{\xi_{\lambda k}-\xi_{\lambda\left(k-Q_{\lambda}\right)}}{Q_{\lambda}} \sum_{k^{\prime}=k+1}^{k+Q_{\lambda}} B_{\lambda k^{\prime}}^{\prime}(t) & \text { if } \xi_{\lambda\left(k-Q_{\lambda}\right)} \leq t<\xi_{\lambda k} \\ \frac{\xi_{\lambda k}-\xi_{\lambda\left(k-Q_{\lambda}\right)}}{Q_{\lambda}} & \text { if } t \geq \xi_{\lambda k} \\ 0 & \text { otherwise. }\end{cases}$

where $B_{\lambda k^{\prime}}^{\prime}$ are splines of order $Q_{\lambda}+1$ defined on the same set of knots. It follows from an analogue of this formula that the normalized B-splines for the frailty density are defined as

$$
\tilde{B}_{u k}(x)=\frac{Q_{u}}{\xi_{u k}-\xi_{u\left(k-Q_{u}\right)}} B_{u k}(x) .
$$

\section{B.2. Moments of a normalized B-spline}

In order to compute $E_{u k}$ denote

$$
M_{n, q, k}=\int_{-\infty}^{\infty} x^{n} \tilde{B}_{q, k}(x) d x
$$

where $\tilde{B}_{q, k}$ is a normalized B-spline of order $q$ with knots $\xi_{-q+1}, \ldots, \xi_{K+q}$. This quantity can be thought of as the $n$-th moment of a random variable whose density is given by a single normalized B-spline. It is easy to show (using integration by parts and a recurrence relation for the derivative of a B-spline), that $M_{n, q, k}$ satisfies the recurrence relation

$$
M_{n, q, k}= \begin{cases}\frac{q}{\xi_{k}-\xi_{k-q}} \cdot \frac{1}{n+1}\left[-M_{n+1, q-1, k-1}+M_{n+1, q-1, k}\right] & \text { if } \xi_{k}-\xi_{k-q}>0 \\ \xi_{k}^{n} & \text { otherwise. }\end{cases}
$$

Given this, the terms $E_{u k}$ can be computed as

$$
E_{u k}=1-M_{1, Q_{u}, k},
$$

using the knots $\xi_{u k}$ corresponding to the normalized B-splines $\tilde{B}_{u k}$.

\section{B.3. Construction of the penalty matrix on the integrated squared second derivative}

The penalty matrix in Section A.3 of the main paper can be computed by two recurrence relations. These relations follow from recurrence relations on B-splines and their derivatives, and integration by parts. Let

$$
\begin{aligned}
f\left(q_{1}, k_{1}, \ell_{1}, q_{2}, k_{2}, \ell_{2}\right) & =\int_{-\infty}^{\infty} B_{q_{1}, k_{1}}^{\left(\ell_{1}\right)}(x) B_{q_{2}, k_{2}}^{\left(\ell_{2}\right)}(x) d x \\
g\left(n, q_{1}, k_{1}, q_{2}, k_{2}\right) & =\int_{-\infty}^{\infty} x^{n} B_{q_{1}, k_{1}}(x) B_{q_{2}, k_{2}}(x) d x
\end{aligned}
$$


where $B_{q, k}^{(\ell)}$ is the $\ell$-th derivative of a spline of order $q$ supported on knots $\left(\xi_{k-q}, \xi_{k}\right)$. Then, the following recurrence relation hold (assume without loss of generality that $\left.\ell_{1} \geq \ell_{2}, q_{1} \geq q_{2}, k_{1} \geq k_{2}\right)$ :

$$
\begin{aligned}
& f\left(q_{1}, k_{1}, \ell_{1}, q_{2}, k_{2}, \ell_{2}\right)= \\
& \begin{cases}0 & \text { if } k_{1}-q_{1} \geq k_{2} \\
g\left(0, q_{1}, k_{1}, q_{2}, k_{2}\right) & \text { if } \ell_{1}=\ell_{2}=0 \\
\frac{q_{1}-1}{\xi_{k_{1}-1}-\xi_{k_{1}-q_{1}}} f\left(q_{1}-1, k_{1}-1, \ell_{1}-1, q_{2}, k_{2}, \ell_{2}\right) & \text { otherwise } \\
\quad-\frac{q_{1}-1}{\xi_{k_{1}}-\xi_{k_{1}-q_{1}+1}} f\left(q_{1}-1, k_{1}, \ell_{1}-1, q_{2}, k_{2}, \ell_{2}\right) & \end{cases} \\
& g\left(n, q_{1}, k_{1}, q_{2}, k_{2}\right)=
\end{aligned}
$$

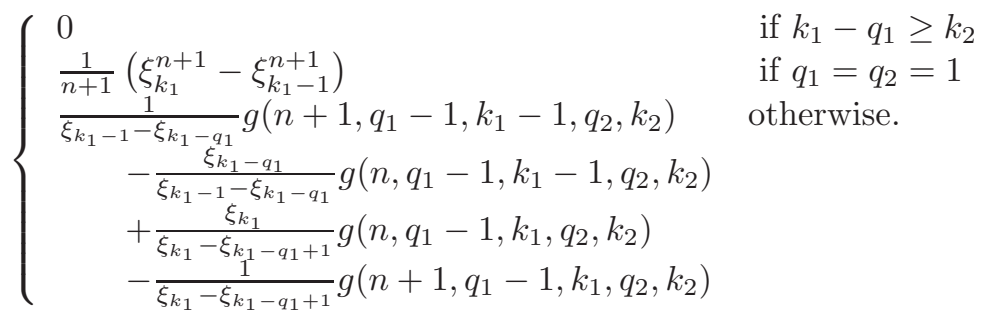

Each entry in the penalty matrix can be separately computed by these recurrence relations.

\section{Appendix C: Gradients and Hessians}

This section includes several gradients and Hessians of the loglikelihoods in Section 2 needed to generate candidates for the MCMC steps. Many of these parameters vary with the iteration $h$ - this dependence is implicit.

The gradient and Hessian of the loglikelihood for the regression coefficients $\boldsymbol{\beta}$ in eq. (2.2) are given by:

$$
\begin{aligned}
\nabla \ell(\boldsymbol{\beta} \mid \ldots) & =\boldsymbol{Z}^{T}\left[\boldsymbol{\delta}-D\left(\boldsymbol{\Lambda}_{0}\right) D\left(e^{\boldsymbol{Z} \boldsymbol{\beta}}\right) \tilde{\boldsymbol{U}}\right]-\frac{1}{\sigma_{\beta}^{2}} \boldsymbol{\beta} \\
\nabla^{2} \ell(\boldsymbol{\beta} \mid \ldots) & =-\boldsymbol{Z}^{T} D\left(\boldsymbol{\Lambda}_{0}\right) D\left(e^{\boldsymbol{Z} \boldsymbol{\beta}}\right) D(\tilde{\boldsymbol{U}}) \boldsymbol{Z}-\frac{1}{\sigma_{\beta}^{2}} \boldsymbol{I}_{p}
\end{aligned}
$$

The gradient and Hessian of the loglikelihood for the baseline hazard spline parameters $\boldsymbol{\theta}_{\lambda}$ in eq. (2.3) are given by:

$$
\begin{aligned}
\nabla \ell\left(\boldsymbol{\theta}_{\lambda} \mid \ldots\right)= & \phi_{\lambda} D\left(e^{\boldsymbol{\theta}_{\lambda}}\right)\left[\boldsymbol{B}_{\lambda}^{T} D\left(\boldsymbol{\lambda}_{0}\right)^{-1} \boldsymbol{\delta}-\boldsymbol{C}_{\lambda}^{T} D\left(e^{\boldsymbol{Z} \boldsymbol{\beta}}\right) \tilde{\boldsymbol{U}}\right]-\frac{1}{2 \sigma_{\lambda}^{2}} \nabla p_{\lambda}\left(\boldsymbol{\theta}_{\lambda}\right) \\
\nabla^{2} \ell\left(\boldsymbol{\theta}_{\lambda} \mid \ldots\right)= & \phi D\left(e^{\boldsymbol{\theta}_{\lambda}}\right)\left[D\left(\boldsymbol{B}_{\lambda}^{T} D\left(\boldsymbol{\lambda}_{0}\right)^{-1} \boldsymbol{\delta}\right)-\phi \boldsymbol{B}_{\lambda}^{T} D(\boldsymbol{\delta}) D\left(\boldsymbol{\lambda}_{0}\right)^{-2} \boldsymbol{B}_{\lambda} D\left(e^{\boldsymbol{\theta}_{\lambda}}\right)\right. \\
& \left.-D\left(\boldsymbol{C}_{\lambda}^{T} D\left(e^{\boldsymbol{Z} \boldsymbol{\beta}}\right) \tilde{\boldsymbol{U}}\right)\right]-\frac{1}{2 \sigma_{\lambda}^{2}} \nabla^{2} p_{\lambda}\left(\boldsymbol{\theta}_{\lambda}\right)
\end{aligned}
$$


The gradient and Hessian of the loglikelihood for the frailty density spline parameters $\boldsymbol{\theta}_{u}$ in eq. (2.4)are given by:

$$
\begin{aligned}
\nabla \ell\left(\boldsymbol{\theta}_{u} \mid \ldots\right)= & \phi D\left(e^{\boldsymbol{\theta}_{u}}\right) \tilde{\boldsymbol{B}}_{u}^{T} D\left(f_{u}\right)^{-1} \mathbf{1}_{m}-2 M \boldsymbol{E}_{u}^{T} e^{\boldsymbol{\theta}_{u}} D\left(\boldsymbol{E}_{u}\right) e^{\boldsymbol{\theta}_{u}}-\frac{1}{2 \sigma_{u}^{2}} \nabla p_{u}\left(\boldsymbol{\theta}_{u}\right) \\
\nabla^{2} \ell\left(\boldsymbol{\theta}_{u} \mid \ldots\right)= & \phi D\left(e^{\boldsymbol{\theta}_{u}}\right)\left[D\left(\tilde{\boldsymbol{B}}_{u}^{T} D\left(f_{u}\right)^{-1} \mathbf{1}_{m}\right)-\phi \tilde{\boldsymbol{B}}_{u}{ }^{T} D\left(f_{u}\right)^{-2} \tilde{\boldsymbol{B}}_{u} D\left(e^{\boldsymbol{\theta}_{u}}\right)\right] \\
& -\frac{1}{2 \sigma_{u}^{2}} \nabla^{2} p_{u}\left(\boldsymbol{\theta}_{u}\right)
\end{aligned}
$$

\section{References}

Cox, M. (1982). Practical spline approximation. In Topics in Numerical Analysis. Lecture Notes in Mathematics 965 79-112. Springer.

DE Boor, C. (2001). A Practical Guide to Splines. Applied Mathematical Sciences 27. Springer.

Denison, D. G. T., Mallick, B. K. and Smith, A. F. M. (1998). Automatic Bayesian curve fitting. Journal of the Royal Statistical Society: Series $B$ (Statistical Methodology) 60 333-350.

Green, P. J. (1995). Reversible Jump Markov Chain Monte Carlo Computation and Bayesian Model Determination. Biometrika 82 711-732. 